
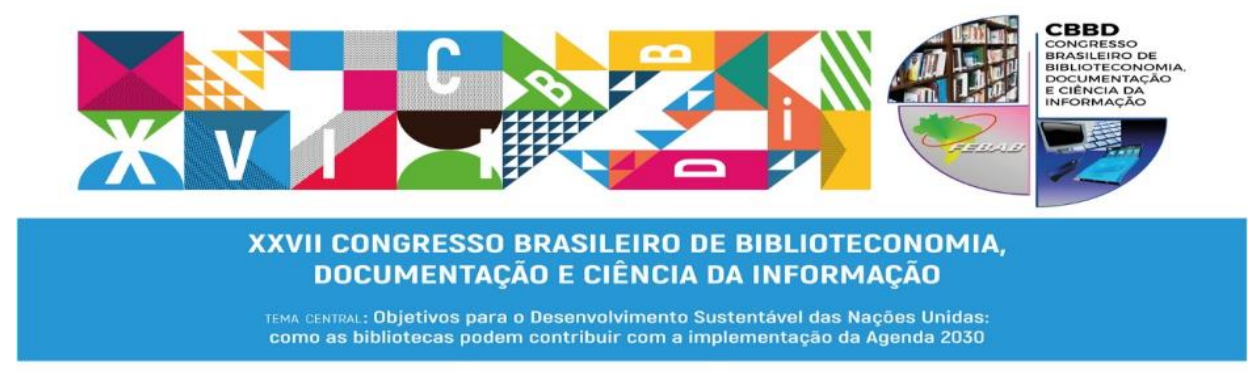

Eixo 1 - Objetivos do Desenvolvimento Sustentável

\title{
BIBLIOTECAS E PESSOAS: interligações positivas na Universidade de Lisboa
}

\section{Luiza Baptista Melo \\ Bibliotecária e Pesquisadora. Faculdade de Medicina Dentária da Universidade de Lisboa, Évora, Portugal. \\ E-mail: luiza.baptista@fmd.ulisboa.pt}

\section{Tatiana Sanches}

Bibliotecária e Pesquisadora.

Faculdade de Psicologia, Universidade de Lisboa, Lisboa, Portugal.

E-mail: tsanches@fpie.ulisboa.pt

\section{Gaspar Matos}

Bibliotecário. Faculdade de Psicologia, Universidade de Lisboa, Lisboa, Portugal.

E-mail: gmmatos@fpie.ulisboa.pt

\section{Patrícia Torres}

Bibliotecária. Faculdade de Medicina Dentária da Universidade de Lisboa, Portugal.

E-mail: patricia.torres@campus.ul.pt

\section{RESUMO}

0 atendimento nas bibliotecas tem vindo a transformarse ao ritmo da tecnologia, supondo-se que a mediação entre informação e utilizadores de bibliotecas poderá estar a perder o seu lugar. No entanto, nas bibliotecas de ensino superior, as mudanças decorrentes da implementação de tecnologias de uma forma ubíqua não retirou a importância do contacto direto dos utentes com os profissionais. 0 presente estudo analisa de que forma as interações dos profissionais têm impacto nos utilizadores. 0 estudo insere-se numa análise mais abrangente e consiste numa avaliação transversal levada a cabo em três instituições de ensino superior, em Portugal, relativa ao atendimento das suas bibliotecas. Para tal foi realizado um inquérito aplicado a uma amostra de utilizadores, alinhado com indicadores das normas ISO 16439, que permitem aferir, entre outros parâmetros, a qualidade do atendimento em bibliotecas, possibilitando medir os fatores que influenciam e afetam os comportamentos dos utilizadores. 0 estudo conclui que as interligações desenvolvidas no atendimento presencial se revelaram positivas e com impactos significativos nos utilizadores.

Keywords: Bibliotecas Universitárias; Atendimento em bibliotecas; Avaliação de bibliotecas; Comportamento dos utilizadores; ISO 16439:2014(E)

\section{LIBRARIES AND PEOPLE: positive connections at the University of Lisbon}

\section{ABSTRACT}

Attendance at libraries has been changing at the pace of technology, supposing that mediation between information and library users may be losing its place. However, in the higher education libraries, the changes resulting from the implementation of technologies in a ubiquitous way did not detract from the importance of the direct contact of the users with the professionals. The present study analyzes how the interactions of professionals have an impact on users. The 


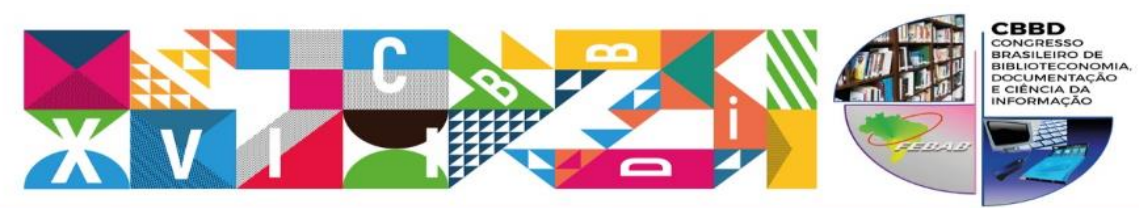

XXVII CONGRESSO BRASILEIRO DE BIBLIOTECONOMIA,

DOCUMENTAÇÃO E CIÊNCIA DA INFORMAÇÃO

TEMA CENIPal: Objetivos para o Desenvolvimento Sustentável das Naçōes Unidas:
como as bibliotecas podem contribuir com a implementaçào da Agenda 2030

study is part of a more comprehensive analysis and consists of a cross-sectional evaluation carried out in three higher education institutions in Portugal, regarding the service of its libraries. For this purpose, a survey was carried out on a sample of users, in line with ISO 16439 standards, which allow us to measure, among other parameters, the quality of service in libraries, making it possible to measure the factors that influence and affect users' behavior. The study concludes that the interconnections developed in face-toface service have proved to be positive and have a significant impact on users.

Keywords: University Libraries; Library attendance; Library assessment; Users' behaviour; ISO 16439:2014(E)

\section{INTRODUÇÃo}

A interação dos utilizadores com os técnicos das bibliotecas académicas permite aproximar uns de outros, estabelecendo o primeiro dos passos que concretizam as práticas de caráter pedagógico para que os utilizadores beneficiem dos recursos disponíveis. Alguns estudos apontam para uma discrepância entre a perceção que os utilizadores têm relativamente à sua autonomia e a verdadeira capacidade de se situarem no espaço e procederem às pesquisas de forma proficiente (por ex., HILBERG \& MEISELWITZ, 2008), relevando daí a necessidade de acederem a práticas ou instruções de caráter educativo, no âmbito da biblioteca.

Para que o conhecimento da biblioteca e dos seus recursos possa chegar ao utilizador de forma mais célere e eficaz é habitual o desenvolvimento de algumas ações de esclarecimento por parte dos técnicos, que se podem revestir de várias modalidades (da visita guiada individual ou em grupo à entrevista de referência, normalmente de caráter individual). Estas interações dos técnicos com o utilizador têm como primeiro objetivo dar a conhecer os espaços, serviços, regras de funcionamento e utilização dos mesmos e os principais procedimentos desenvolvidos na biblioteca. Muitas vezes esta conversa é dirigida a não frequentadores do espaço, precisamente com o objetivo de fazer com que o venham a frequentar e utilizar. Importa pois, desde logo, estabelecer uma empatia entre quem recebe e quem visita, e ao mesmo tempo transparecer o 


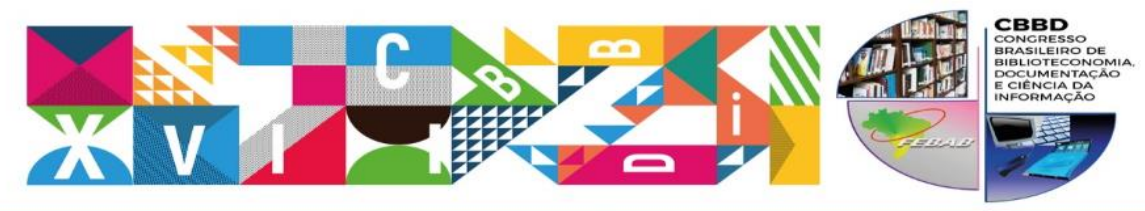

XXVII CONGRESSO BRASILEIRO DE BIBLIOTECONOMIA, DOCUMENTAÇÃO E CIÊNCIA DA INFORMAÇÃO

TEMA CENIPal: Objetivos para o Desenvolvimento Sustentável das Naçôes Unidas:
como as bibliotecas podem contribuir com a implementaçào da Agenda 2030

compromisso e a identidade institucional que o profissional tem com o serviço que representa (OBOLER, 1977: 148).

Numa fase posterior, conhecido que é o espaço e a sua organização, conhecidas as regras de funcionamento e os procedimentos de rotina para a utilização dos recursos documentais, os utilizadores podem frequentar os serviços, previsivelmente, com maior benefício. Contudo, nem sempre encontram o que procuram. É nesta situação que se dirigem aos profissionais da biblioteca.

Nesta altura sucede a entrevista de referência, um momento iminentemente pedagógico, em que se põe em curso um diálogo entre bibliotecário e utilizador, com vista a esclarecer e focar a pesquisa, de forma a obter a informação mais adequada e pertinente para o utilizador. Fernández y Fernández-Cuesta (2005: 51) descreve este processo como um trabalho intelectual e científico de mediação, referindo que para o mediador o principal trabalho é o de escutar, captar ideias, perguntar quem, como e onde se investiga determinado assunto, colocar especialistas em contacto, relacionar fontes e investigadores e, entre outras, conhecer tendências, estabelecer relações, cultivar conversas para que delas germinem ideias.

Este processo de facilitação é amplamente reconhecido como indispensável, quer para as bibliotecas físicas, quer virtuais, permitindo uma aproximação intermediada entre o utilizador e os recursos informativos. É interessante sublinhar a ideia de que a interação neste momento é, além de pedagógica, de útil partilha de conhecimento das necessidades, de forma recíproca, isto é, tanto o aluno expõe o que pretende, como o bibliotecário obtém o conhecimento acerca do que precisa para prover a biblioteca de informação adequada para os seus utilizadores, ideia aliás já aflorada por outros bibliotecários (KING, 1977: 39).

As linhas de orientação preconizadas para esta interação, e emanadas da American Library Association (ALA), constituem-se como uma moldura conceptual e uma ética de serviço que ajuda os profissionais de informação a definirem padrões de qualidade neste atendimento específico e especializado. No documento orientador Guidelines for Behavioral Performance of Reference and Information Service Providers, é afirmado (ALA, 2008) que em todas as formas de serviços de referência, o sucesso da transação é medido não apenas pela informação transmitida, mas também pelo impacto positivo ou negativo 


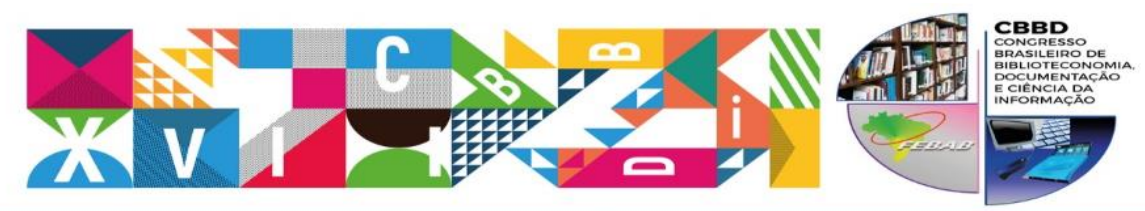

XXVII CONGRESSO BRASILEIRO DE BIBLIOTECONOMIA, DOCUMENTAÇÃO E CIÊNCIA DA INFORMAÇÃO

TEMA CENIPal: Objetivos para o Desenvolvimento Sustentável das Naçōes Unidas:
como as bibliotecas podem contribuir com a implementaçào da Agenda 2030

da interação. 0 comportamento positivo ou negativo do membro da equipa de referência (conforme observado pelo utilizador) torna-se um fator significativo no sucesso ou falha percebida.

A entrevista de referência significa pois uma experiência positiva ou negativa de acordo com a maior atenção e disponibilidade do bibliotecário para esta interação, já que, segundo os estudos citados pela ALA (2008), um maior empenho por parte dos técnicos resulta numa maior satisfação por parte dos utilizadores, até mesmo quando não encontram aquilo que procuram. Este momento obedece a etapas muito bem estruturadas, em torno de cinco eixos principais: a acessibilidade dos técnicos; o interesse demonstrado; a forma de escutar/ perguntar; a realização da pesquisa; e o seguimento ou follow up. De facto, nesta interação personalizada, é muito importante estabelecer o contacto visual e manter o interesse ao longo de todo o processo, averiguando se as necessidades de informação do utilizador ficaram satisfeitas no final. Conclui-se, pela revisão da literatura, que a entrevista de referência é, pelas suas características intrínsecas de apoio personalizado, uma prática muito bem acolhida pelos utilizadores, podendo ser um excelente indicador da qualidade percebida dos serviços.

\section{MÉTODO DA PESQUISA}

Este estudo recorre a análises quantitativa e qualitativa para descrever e explicar como as interações entre profissionais e utilizadores das bibliotecas universitárias podem afetar o comportamento destes últimos perante a informação. 0 estudo de caso envolve um universo de cerca de 2760 potenciais utilizadores de duas bibliotecas académicas da Universidade de Lisboa. 0 universo é constituído por docentes, investigadores, estudantes e funcionários da Faculdade de Medicina Dentária (FMD), da Faculdade de Psicologia (FP) e do Instituto de Educação (IE).

Os dados têm origem num estudo mais alargado de Melo, Matos, Torres e Sanches (2017) e foram obtidos por um inquérito via Internet e em suporte papel, que foi criado de acordo com os seguintes objetivos - identificar o utilizador, avaliar o impacto da biblioteca na instituição, o impacto das instalações da biblioteca no bem-estar do 

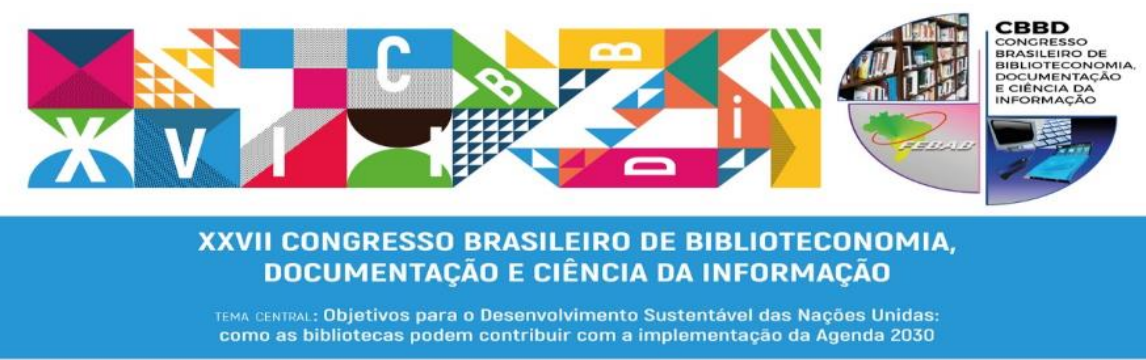

utilizador, o sucesso nos processos de investigação, aprendizagem e desempenho profissional e, finalmente, estimar a qualidade do serviço prestado pelos funcionários. Essas questões foram convertidas em indicadores de impacto definidos pelas normas internacionais ISO 16439: 2014(E) - Information and documentation -- Methods and procedures for assessing the impact of libraries (ISO, 2014), nomeadamente: Obtenção de informação útil para o estudo e investigação; Grau da empatia dos funcionários; Utilidade das respostas dos funcionários.

Tabela 1. Objetivos da avaliação e indicadores da ISO 16439:2014(E) que alicerçaram a criação do questionário

\begin{tabular}{|c|c|c|}
\hline Objetivo de avaliação & Indicador & \\
\hline $\begin{array}{l}\text { Instituição a que } \\
\text { pertencem e categoria }\end{array}$ & $\begin{array}{c}\text { IS016439:2014(6.2.2.3) - Estatística de } \\
\text { utilizador }\end{array}$ & $*$ \\
\hline $\begin{array}{l}\text { Identificação do género } \\
\text { do utilizador }\end{array}$ & $\begin{array}{c}\text { IS016439:2014(6.2.2.3) - Estatística de } \\
\text { utilizador }\end{array}$ & I.1 \\
\hline Uso da biblioteca & \multirow{3}{*}{$\begin{array}{c}\text { IS016439:2014(6.3.2.2) - Indicador de } \\
\text { impacto } \\
\text { Desempenho da biblioteca }\end{array}$} & I.2 \\
\hline Frequência de utilização & & I.3 \\
\hline $\begin{array}{l}\text { Objetivo do uso da } \\
\text { biblioteca }\end{array}$ & & I.4 \\
\hline $\begin{array}{l}\text { Segurança das } \\
\text { instalações }\end{array}$ & \multirow{5}{*}{$\begin{array}{c}\text { Efeitos do impacto da biblioteca } \\
\text { ISO16439:2014(4.4.2.4) - impacto no } \\
\text { bem-estar individual }\end{array}$} & I.5 \\
\hline $\begin{array}{l}\text { Grau de conforto das } \\
\text { instalações }\end{array}$ & & I.6 \\
\hline $\begin{array}{l}\text { Ambiente favorável ao } \\
\text { estudo }\end{array}$ & & I.7 \\
\hline Sentimento de igualdade & & I.8 \\
\hline Sentimento de pertença & & I.9 \\
\hline $\begin{array}{l}\text { Obtenção de informação } \\
\text { útil para o estudo } \\
\text { e investigação }\end{array}$ & $\begin{array}{c}\text { Efeitos do impacto da biblioteca } \\
\text { ISO16439:2014(4.4.2.3) - Indicador do } \\
\text { maior sucesso na pesquisa, estudo e carreira }\end{array}$ & I.10 \\
\hline $\begin{array}{l}\text { Grau da empatia dos } \\
\text { funcionários }\end{array}$ & \multirow{3}{*}{$\begin{array}{l}\text { Desempenho da biblioteca } \\
\text { ISO16439:2014(6.3.2.3) - } \\
\text { Qualidade dos serviços prestados }\end{array}$} & I.11 \\
\hline $\begin{array}{l}\text { Utilidade das respostas } \\
\text { dos funcionários }\end{array}$ & & I.12 \\
\hline Sugestões & & \\
\hline
\end{tabular}

Fonte: Dados da amostra

Os dados foram recolhidos por inquérito, via Internet nas instalações das bibliotecas da FMD e da FP-IE, e em suporte papel nas clínicas da FMD. O período de 

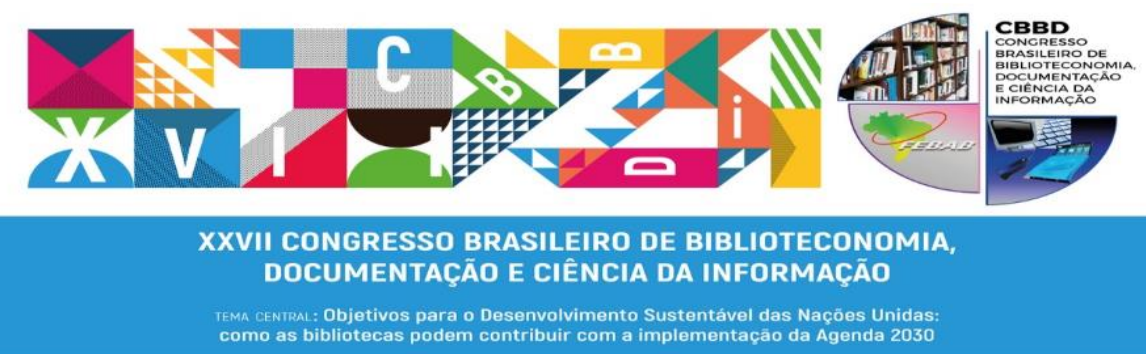

recolha de dados decorreu de Dezembro de 2016 a Janeiro de 2017. A investigação desenvolveu-se através de uma análise dos resultados baseada na estatística descritiva (frequências absolutas, frequências relativas e tabelas de contingência) e inferência. Com apoio das técnicas de benchmarking, processo que observa boas práticas que conduzem a melhores desempenhos, indicam-se algumas soluções para futuramente serem implementadas nestas bibliotecas académicas.

Para o presente estudo foram selecionadas as questões concretas que envolvem as interações dos profissionais com os utilizadores (relativas a Efeitos do impacto da biblioteca IS016439:2014 (4.4.2.3) - Indicador do maior sucesso na pesquisa, estudo e carreira; Desempenho da biblioteca IS016439:2014 (6.3.2.3) - Qualidade dos serviços prestados).

\section{RESULTADOS}

Este estudo decorreu em duas bibliotecas de três escolas da Universidade de Lisboa, respetivamente, na Faculdade de Medicina Dentária (FMDUL) e na Faculdade de Psicologia e Instituto de Educação (FP-IE). 0 universo académico investigado é constituído uma população de cerca de 600 estudantes, 128 docentes e investigadores e 63 funcionários da FMDUL e 959 estudantes, 58 docentes, 5 investigadores e 27 funcionários da FP e 827 estudantes, 61 docentes, 4 investigadores e 27 funcionários do IE. As Tabelas 2 e 3 apresentam o universo e a amostra:

Tabela 2. Constituição da população em estudo

\begin{tabular}{|lrrrr|}
\hline \multicolumn{5}{c}{ Universo } \\
Tipo & FMD & FP & IE & \multicolumn{1}{c|}{ Totais } \\
\hline Docentes & 128 & 58 & 61 & 247 \\
\hline Investigadores & 0 & 5 & 4 & 9 \\
\hline Estudantes & 600 & 959 & 827 & 2386 \\
\hline Funcionários & 63 & 27 & 27 & 117 \\
\hline Totais & 791 & 1049 & 919 & 2759 \\
\hline Total & & & & $\mathbf{2 7 5 9}$ \\
\hline
\end{tabular}

Fonte: Dados da Pesquisa 

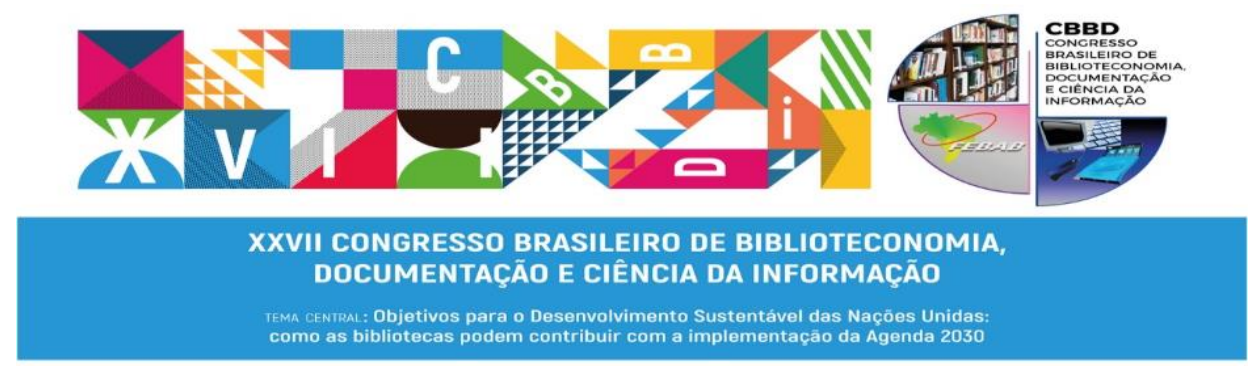

Tabela 3. Constituição da amostra em estudo

\begin{tabular}{|c|c|c|c|c|}
\hline \multicolumn{5}{|c|}{ Amostra } \\
\hline & FMD & $\mathrm{FP}$ & IE & Outros \\
\hline No. Respostas & 275 & 84 & 24 & 5 \\
\hline & $71 \%$ & $22 \%$ & $6,10 \%$ & $1,30 \%$ \\
\hline
\end{tabular}

Fonte: Dados da Pesquisa

Tabela 4. Identificação do género do utilizador

\begin{tabular}{|c|c|c|c|}
\hline Indicador & Género & $\%$ & $N^{o}$ \\
\hline \multirow{3}{*}{ I.1 } & Feminino & $75.86 \%$ & 286 \\
\hline & Masculino & $24.14 \%$ & 91 \\
\hline & Total & $100 \%$ & 377 \\
\hline
\end{tabular}

Fonte: Dados da Pesquisa

Em síntese, o universo investigado é constituído por cerca de 600 estudantes, 128 docentes e investigadores e 63 funcionários da FMDUL e 959 estudantes, 58 docentes, 5 investigadores e 27 funcionários da FP e 827 estudantes, 61 docentes, 4 investigadores e 27 funcionários do IE. Relativamente à amostra considerada, foram obtidas um total de 388 respostas totais. Em linha com a tendência nacional, obtiveram-se cerca de um quarto de respostas de pessoas do género masculino e três quartos do género feminino.

Como já referido, a investigação implementou conhecimentos da estatística descritiva (frequências absolutas, frequências relativas e tabelas de contingência) e inferência na realização da análise dos dados obtidos numa amostra de cerca de $14 \%$ da população académica em estudo. No decorrer da análise dos dados observaram-se alguns problemas resultantes da distribuição e representatividade discrepante nas duas bibliotecas das instituições analisadas. Ainda assim, obtiveram-se conclusões úteis e desenvolveu-se uma reflexão teórica baseada nos conceitos de atendimento, especificamente aquele que é realizado presencialmente em bibliotecas, no contexto do ensino superior. Analisam-se também as respostas que podem ser associadas ao comportamento de docentes, estudantes, investigadores, funcionários e profissionais relativamente à informação que utilizam nestes serviços e obtida particularmente por via desse mesmo atendimento. 

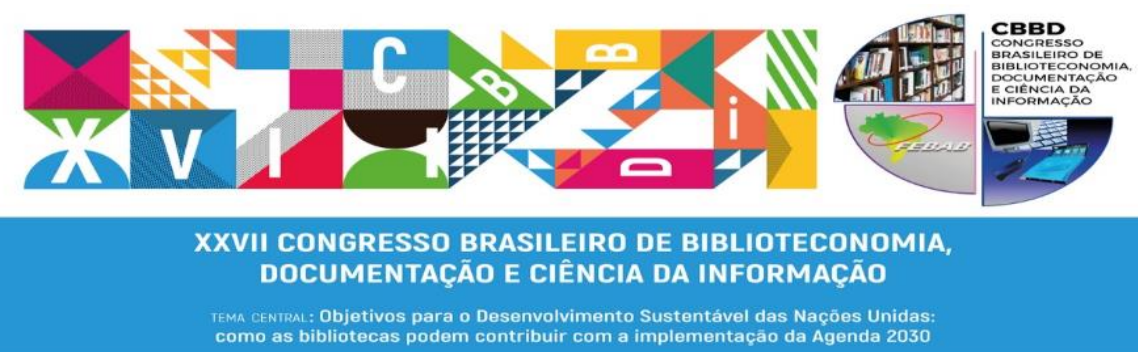

Os resultados globais surgem nas tabelas seguintes, onde se encontram elencadas as questões do inquérito com as respetivas respostas, apresentando-se os dados relativos aos resultados obtidos, de onde posteriormente extraímos as questões específicas relativas ao atendimento. Foram portanto excluídas, deste estudo, as respostas relacionadas com a Segurança das instalações, Grau de conforto das instalações, Ambiente favorável ao estudo, Sentimento de igualdade e Sentimento de pertença.

Note-se ainda que as respostas totais não coincidem de questão para questão, pois alguns inquiridos não responderam a todas as questões.

\section{Gráfico 1. Uso da biblioteca}

\section{Frequenta a biblioteca?}

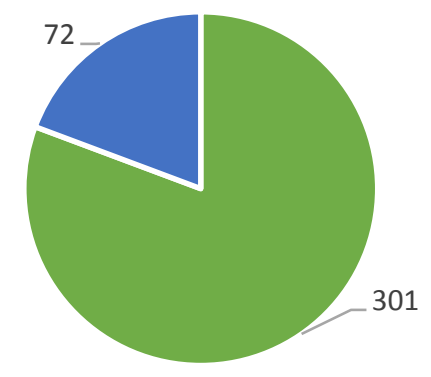

- Sim $80.70 \%$ - Não $19.30 \%$

Fonte: Dados da Pesquisa

Da amostra recolhida observa-se uma frequência habitual declarada por $80.70 \%$ dos usuários, em confronto com os $19.30 \%$ que declaram não frequentar. 

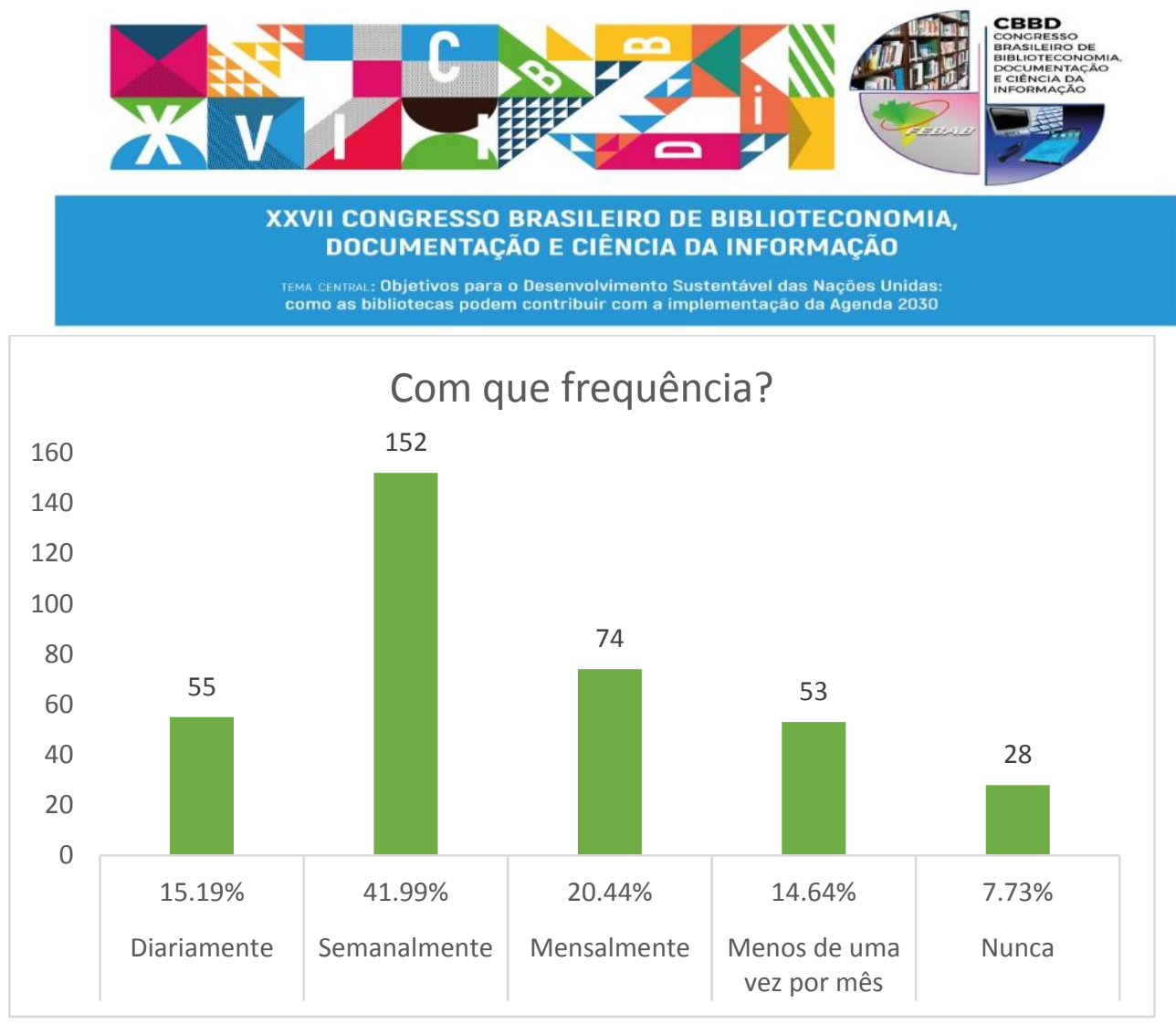

Fonte: Dados da Pesquisa

Quanto à utilização, verificamos uma assiduidade significativa nos usuários que declaram vir à biblioteca, destacando-se os que visitam semanalmente as instalações.

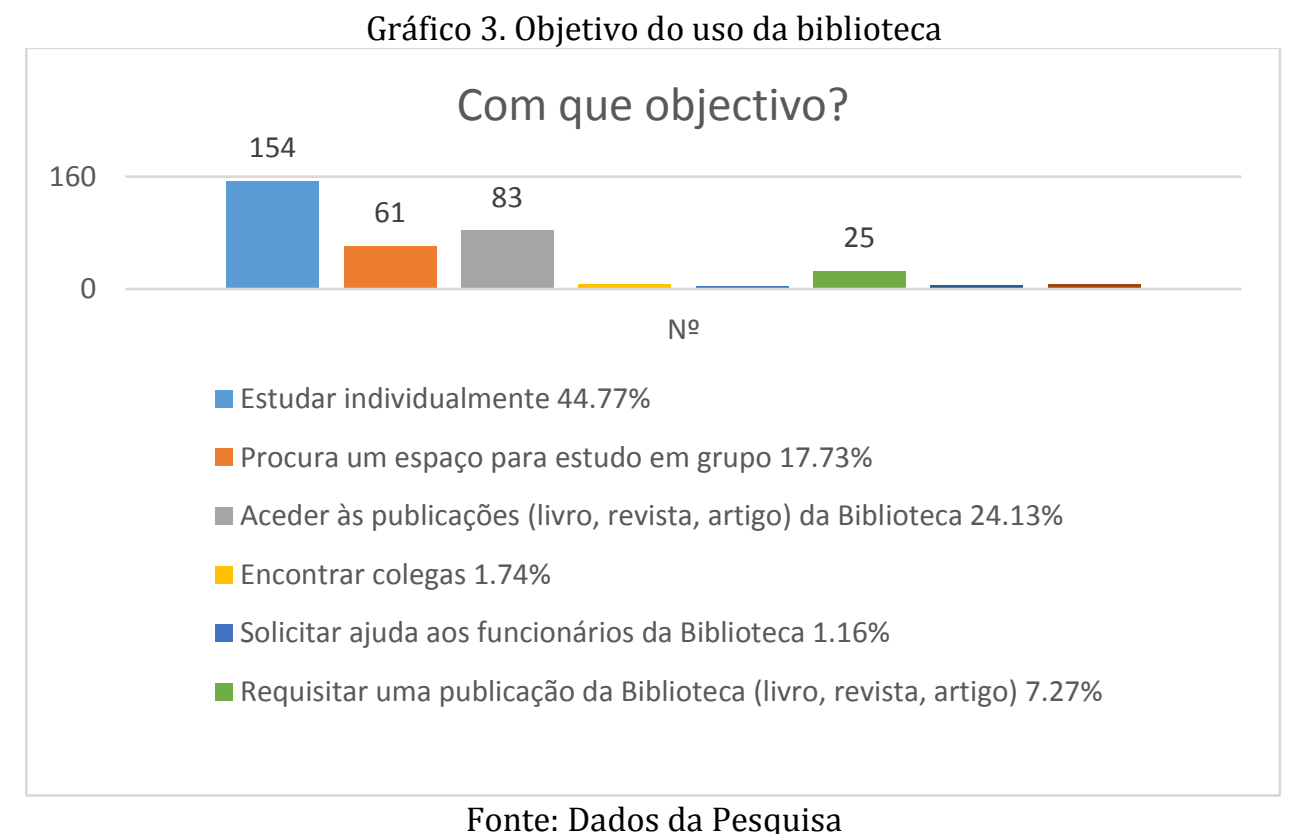

Fonte: Dados da Pesquisa

Relativamente à utilização da biblioteca, cerca de metade aponta o estudo individual como principal objetivo desta frequência, seguindo-se o acesso à informação livros, revistas, artigos, entre outros. É importante sublinhar este aspeto, visto que a 


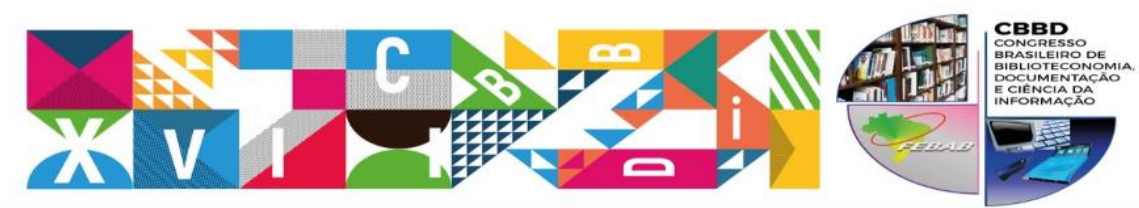

XXVII CONGRESSO BRASILEIRO DE BIBLIOTECONOMIA, DOCUMENTAÇÃO E CIÊNCIA DA INFORMAÇÃO

TEMA CENIPAL : Objetivos para o Desenvolvimento Sustentável das Naçōes Unidas:
Como as bibliotecas podem contribuir com a implementaça da Agenda 2030

biblioteca continua a ser o local onde a informação é encontrada de uma forma fiável, pois é globalmente validada e está organizada de modo a facilitar a sua consulta direta. Finalmente, das restantes opções, destaca-se ainda o estudo em grupo como significativo. A biblioteca é também considerada um local de trabalho colaborativo.

Gráfico 4. Obtenção de informação útil para o estudo e investigação

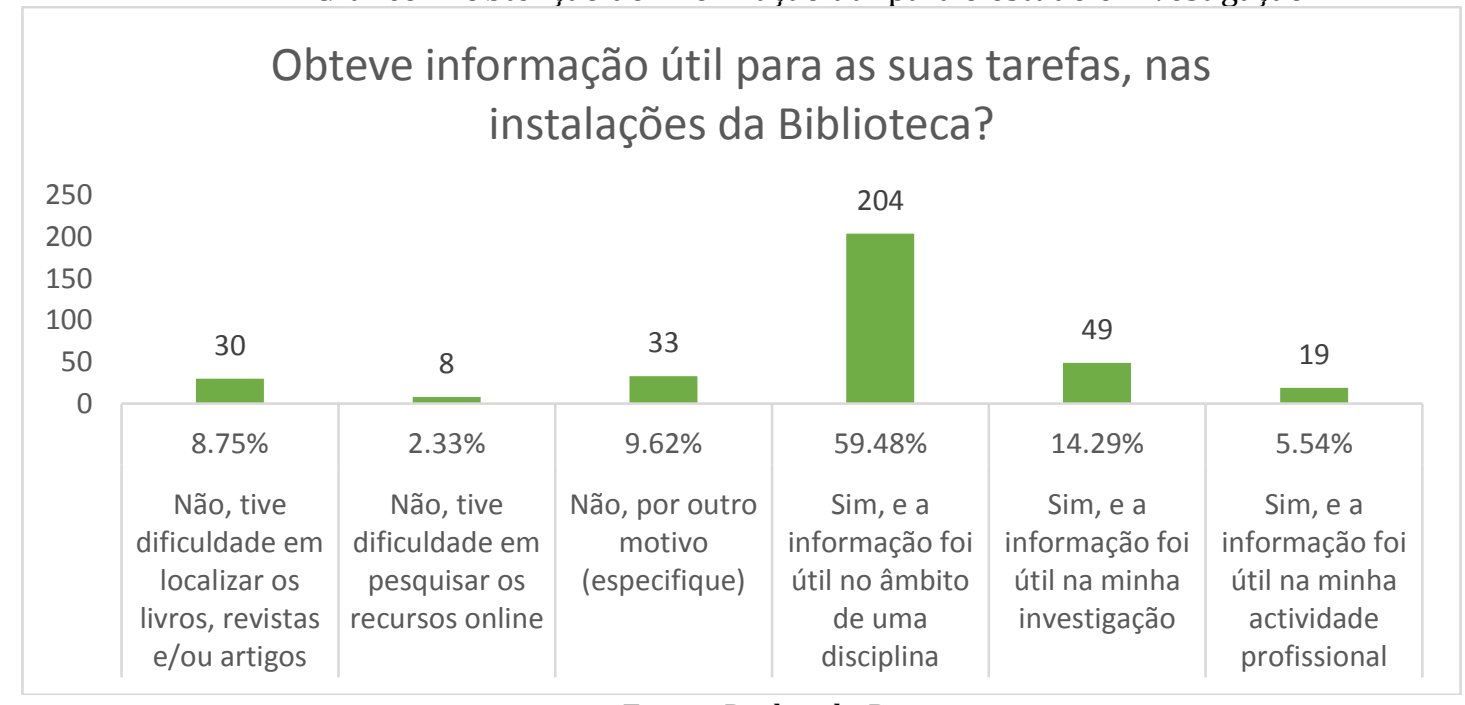

Fonte: Dados da Pesquisa

Nas respostas obtidas à questão sobre a utilidade da informação, os respondentes foram claros, afirmando que sim na sua maioria. Especificamente destaca-se que a utilização desta informação, neste âmbito particular, é sobretudo tendo em vista o cumprimento de objetivos académicos.

Gráfico 5. Grau de empatia dos funcionários

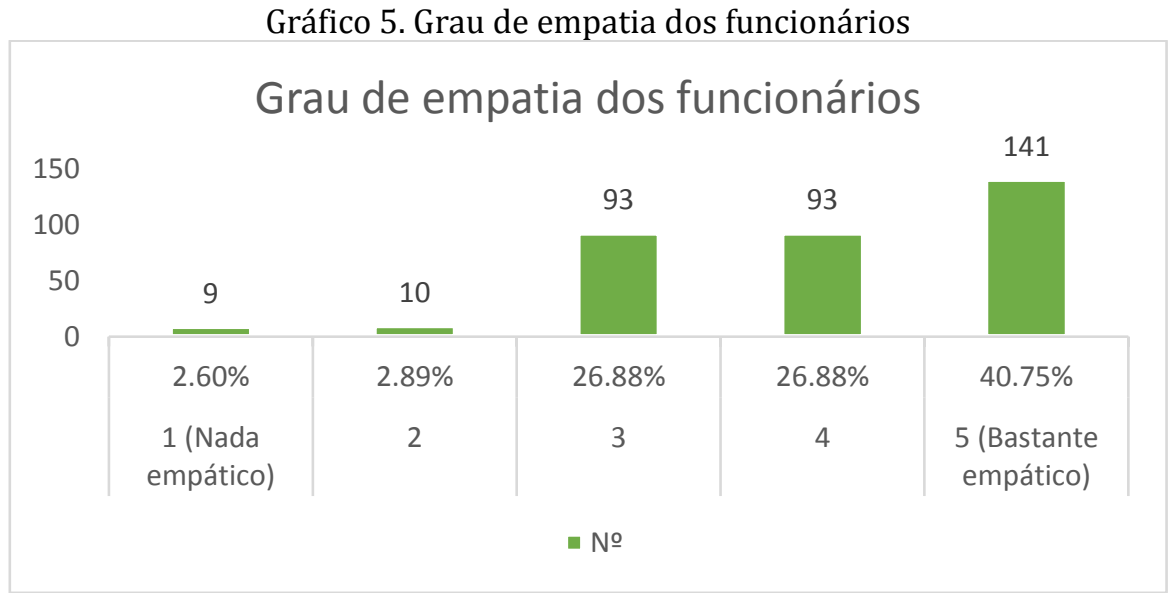

Fonte: Elaborado pelos autores 


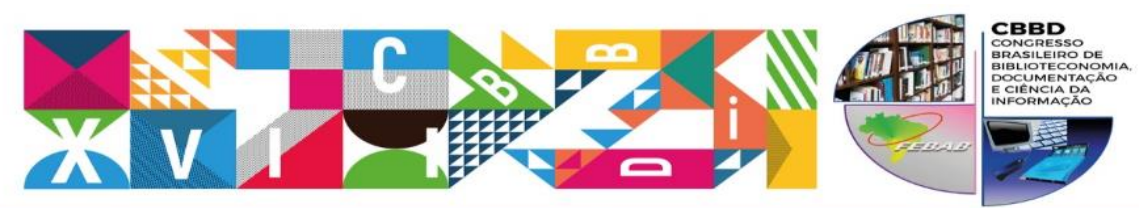

XXVII CONGRESSO BRASILEIRO DE BIBLIOTECONOMIA, DOCUMENTAÇÃO E CIÊNCIA DA INFORMAÇÃO

TEMA CENIRal: Objetivos para o Desenvolvimento Sustentável das Naçôes Unidas:
como as bibliotecas podem contribuir com a implementaçăo da Agenda 2030

Quanto ao grau de empatia, é residual a taxa de respostas menos positivas. Mais de 90\% posicionam as suas respostas no empático, muito empático ou bastante empático para avaliar os funcionários do atendimento.

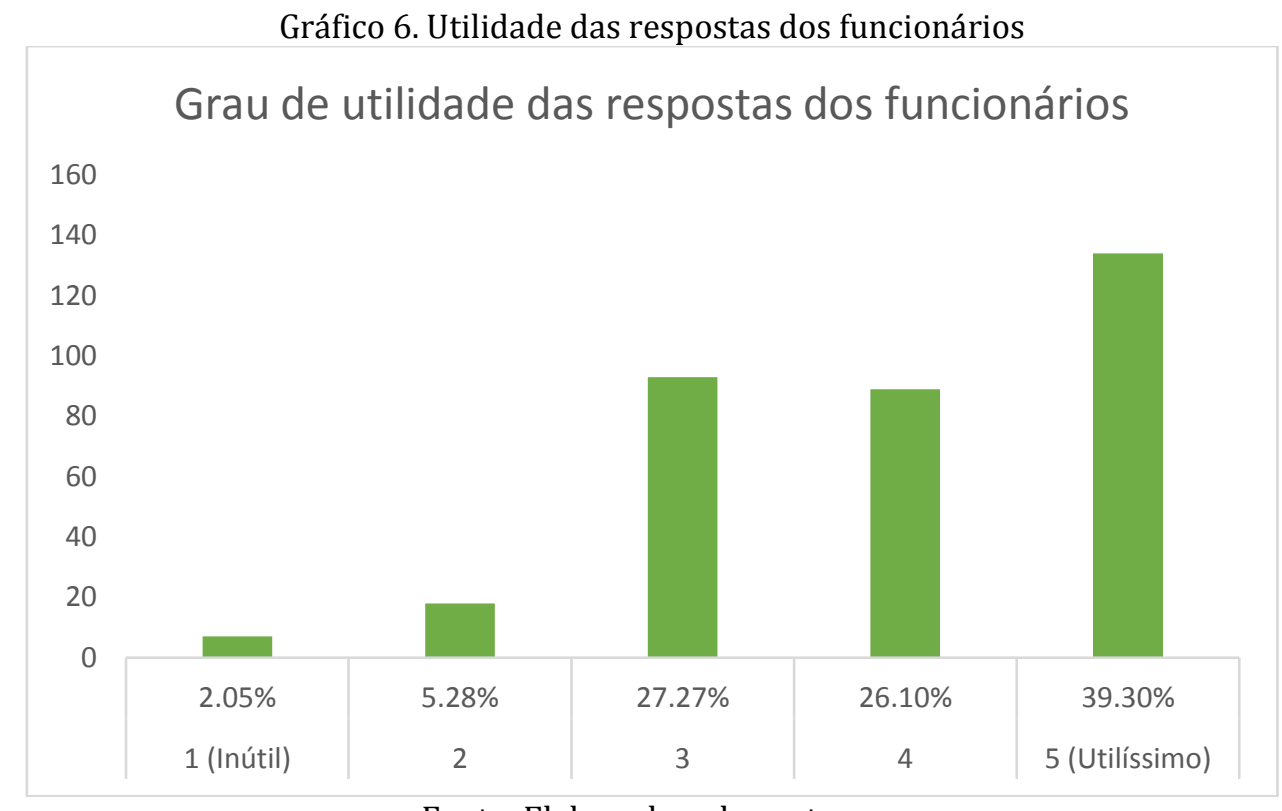

Fonte: Elaborado pelos autores

Finalmente, no que concerne à utilidade das respostas dos funcionários, encontramos a mesma tendência muito positiva, destacando-se mesmo, no topo das respostas uma classificação de utilíssima para avaliar a informação fornecida pelos técnicos.

Em resumo, no que se relaciona com o género, dos resultados obtidos, observa-se neste contexto que existe uma tendência semelhante ao universo dos alunos de ensino superior português, (feminino 55\% - masculino 45\%, aproximadamente, no panorama nacional; $75 \%$ - 25\% nas nossas respostas).

Quanto à taxa de frequência dos respondentes, esta encontra-se nos $80 \%$ e é maioritariamente semanal, mensal e diária (estes três itens com mais de $75 \%$ das respostas), isto é, os respondentes são relativamente assíduos.

Quanto aos objetivos, $80 \%$ reconhecem utilidade na Biblioteca, ao afirmar encontrar lá os recursos de que necessitam. Esta taxa de satisfação tão alta demonstra que 


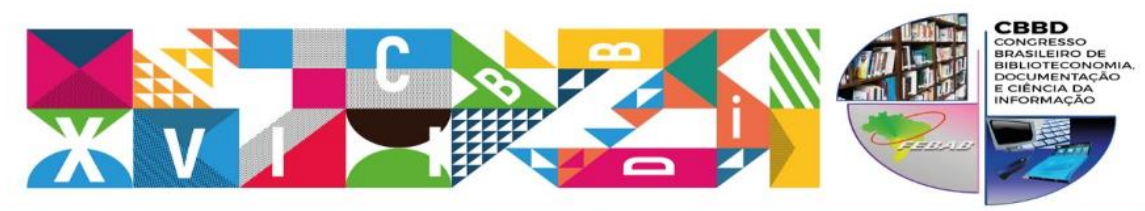

XXVII CONGRESSO BRASILEIRO DE BIBLIOTECONOMIA, DOCUMENTAÇÃ̃O E CIÊNCIA DA INFORMAÇĀO

IEMA cENIRAL: Objetivos para o Desenvolvimento Sustentável das Naçöes Unidas:
como as bibliotecas podem contribuir com a implementaçăo da Agenda 2030

os recursos e coleções disponíveis são adequados às necessidades dos usuários, porém, não se trata apenas disso. A interação existente, nomeadamente porque os funcionários esclarecem as técnicas de pesquisa mais eficazes e elucidam sobre o que é mais adequado para cada necessidade de informação, torna a experiência de pesquisar mais satisfatória para estes destinatários.

O grau de empatia dos funcionários corrobora esta ideia. Para com os inquiridos, a empatia dos funcionários que se ocupam do atendimento é bastante elevada (52\% para Empático e Muito Empático e 40\% para Bastante Empático) e, ao mesmo tempo, as respostas dos funcionários são consideradas Úteis ou Muito Úteis por mais de 53\% dos inquiridos, e Bastante Úteis por quase 40\% dos indivíduos.

\section{DISCUSSÃo}

Em suma pode-se afirmar que se valida, nas Bibliotecas analisadas (e nomeadamente cruzando os indicadores Obteve Informação Útil, Grau de Empatia e Utilidade das Respostas dos Funcionários) a tese que dá título a este artigo, ou seja, que as interações entre técnicos e utilizadores têm um reflexo positivo não só no desempenho académico dos segundos mas também que, obviamente, refletem a competência e utilidade dos primeiros, confirmando-se as ideias expressas na revisão da literatura.

Na verdade, é muito importante compreender que o sucesso e satisfação obtidos na busca de informação na biblioteca é medido não apenas pela obtenção de informação adequada, mas essencialmente pela forma como o atendimento é realizado. Isto significa que o comportamento positivo ou negativo do membro da equipa de referência (conforme observado pelo utilizador) torna-se um fator significativo no sucesso ou falha percebida. Daí ser tão importante consciencializar o técnico da importância de um bom atendimento.

\section{CONSIDERAÇÕES FINAIS}

A investigação foi baseada em vários indicadores das normas internacionais IS016439(E), cujo instrumento de aferição correspondeu bem às necessidades do estudo 


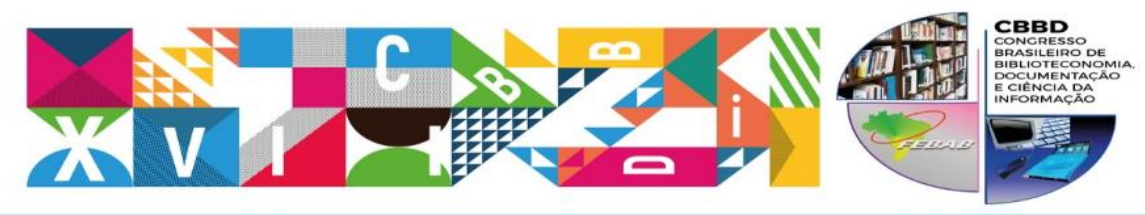

XXVII CONGRESSO BRASILEIRO DE BIBLIOTECONOMIA, DOCUMENTAÇÃ̃O E CIÊNCIA DA INFORMAÇĀO

TEMA CENIRAL: Objetivos para o Desenvolvimento Sustentável das Naçöes Unidas:
como as bibliotecas podem contribuir com a implementaça da Agenda 2030

concretizado. As evidências observadas suportam a hipótese de que o atendimento das bibliotecas académicas influencia os comportamentos de pesquisa e a perceção de utilidade de informação por parte dos docentes, investigadores e estudantes, desempenhando um papel fundamental no processo de ensino, aprendizagem e investigação e revelando interações positivas no caso em análise. A análise da biblioteca académica como um espaço que propícia impacto e valor, melhorando o processo de ensino e aprendizagem é confirmada nas várias escolas de ensino superior estudadas, independentemente, de um ensino mais teórico ou mais prático.

De facto, ao verificarmos os resultados da avaliação demonstrados por este projeto, confirma-se a ideia de partida, verificada na revisão da literatura: é que as conexões positivas entre os usuários e os atendedores validam a qualidade dos serviços. Os recursos da biblioteca valorizam a aprendizagem dos alunos e o seu sucesso académico, designadamente quando estes são fornecidos através de um serviço qualificado. Assim, pode constatar-se que os serviços de apoio à pesquisa na biblioteca amplificam a aprendizagem dos alunos. 0 contributo das bibliotecas é também reconhecido como fator de retenção dos alunos, isto é, por terem formação e acompanhamento na biblioteca, é mais provável que permaneçam no sistema de ensino e prossigam para a conclusão dos seus estudos.

De referir ainda que, por haver esta ligação de permanente disponibilidade para o atendimento, se acrescenta valor à experiência académica, promovendo-se o relacionamento e o envolvimento dos alunos entre pares e com a sua comunidade académica. Podemos assim afirmar que os serviços de referência e de atendimento, no caso em análise, se revelam importantes, deduzindo-se daqui uma relação positiva com a aprendizagem dos alunos e o seu sucesso académico.

$\mathrm{Na}$ atualidade, e com a emergência das tecnologias nas bibliotecas académicas, a informação é amplamente disseminada online. Neste contexto a mediação dos técnicos, não perdeu validade, pelo contrário: essa intervenção acrescenta valor à recuperação de informação (BANDYOPADHYAY \& BOYD-BYRNES, 2016; PATERSON, 2016), que se traduz na obtenção mais pertinente e mais rápida da informação científica, necessária ao bom desenvolvimento das atividades de aprendizagem, ensino e pesquisa. 


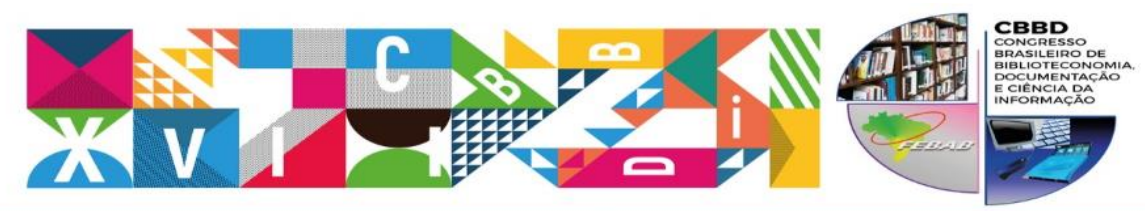

XXVII CONGRESSO BRASILEIRO DE BIBLIOTECONOMIA, DOCUMENTAÇÃO E CIÊNCIA DA INFORMAÇÃO

TEMA CENIPal: Objetivos para o Desenvolvimento Sustentável das Naçôes Unidas:
como as bibliotecas podem contribuir com a implementaçào da Agenda 2030

Compreender como as pessoas destas comunidades em particular usam e se apropriam da informação existente na biblioteca é importante para podermos atuar no futuro. É igualmente importante entender as competências que os usuários têm para encontrar informação útil, bem como aquelas que os bibliotecários necessitam desenvolver para fomentar essas competências com base em bons relacionamentos. Por este motivo, estudos de caso como este, com várias bibliotecas da mesma Universidade, ou com outras instituições de ensino superior nacionais ou estrangeiras devem ser fomentados para a análise de boas práticas e realização de melhorias concretas.

Para o futuro seria interessante realizar-se uma extrapolação mais sustentada, partindo de um universo de análise mais alargado. É útil prosseguir com uma consciencialização dos profissionais exercem funções de front office, pois estes têm impacto direto no comportamento dos utilizadores, permitindo, ao investirmos nestas vertentes, obter benefícios para todas as partes interessadas.

\section{REFERÊNCIAS}

ALA. Guidelines for Behavioral Performance of Reference and Information Service Providers, Chicago: Reference and User Services Association, American Library Association. 2008. Acesso em: http://www.ala.org/rusa/resources/guidelines/guidelinesbehavioral

BANDAYOPADHAY A., BOYD-BYRNE M. K. Is the need for mediated reference service in academic libraries fading away in the digital environment?, Reference Services Review, 44(4) 2016, pp.596-626,Acesso em: https://doi.org/10.1108/RSR-02-2016-0012

FERNÁNDEZ Y FERNÁNDEZ-CUESTA, P. Bibliotecas y personas: hacia un nuevo enfoque en biblioteconomía. Gijón: Trea. 2005.

HILBERG, J. S. \& MEISELWITZ, G. Undergraduate fluency with information and communication technology: perceptions and reality. In Association for Computing Machinery. Information technology education: Proceedings of the 9th ACM SIGITE conference, (SIGITE '08), 2008, pp.5-10. DOI:10.1145/1414558.1414562

ISO - International Organization for Standardization. Information and documentation Methods and procedures for assessing the impact of libraries: Information et documentation Méthodes et procédures pour évaluer l'impact des bibliothèques. Genebra, 2014. Disponível em: https://www.iso.org/obp/ui/\#iso:std:iso:16439:ed-1:v1:en

KING T. A. Serviços para o leitor em bibliotecas. In: Cadernos de biblioteconomia, arquivística e documentação. Coimbra, 13 (1) Jan.-Jun. 1977, pp. 34-41. 

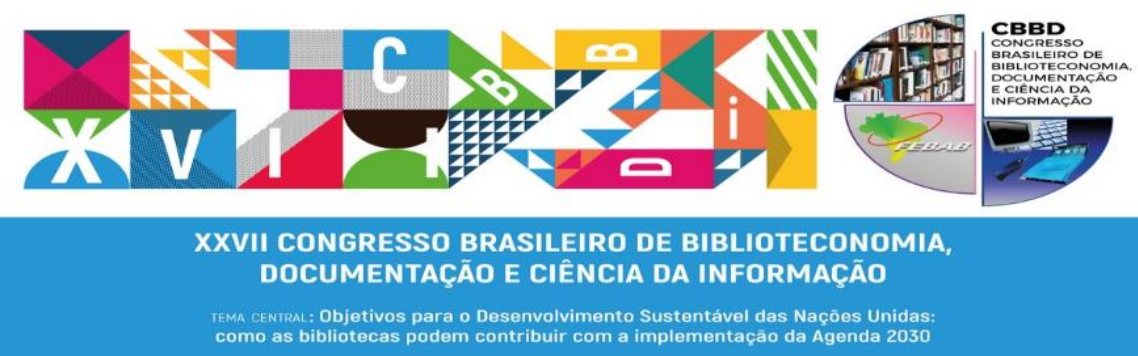

MELO, L.B.; MATOS, G.; TORRES, P.; SANCHES, T. Assessing the impact of academic library spaces on users' behavior with the IS016439:2014(E). In 9th Qualitative and Quantitative Methods in Libraries International Conference, Limerick, Ireland, 23-26 May 2017.

OBOLER, E. M. Ideas and the university library: essays of an unorthodox academic librarian, London, Greenwood, 1977.

PATERSON, A. After the desk: reference service in a changing information landscape. In J. HagenMcIntosh. Information and data literacy: the role of the library. Oakville: Apple Academic Press, pp. 67-76, 2016.

Agências financiadoras - Este trabalho é co-financiado pela União Europeia através do Fundo Europeu de Desenvolvimento Regional, enquadrado no COMPETE 2020 (Programa Operacional da Competitividade e Internacionalização) através do projeto CIDEHUS - UID/HIS/00057/2013 (POCI-01-0145-FEDER-007702) 